\title{
Orbital Decay of the PSR J0045-7319 Binary System: Age of Radio Pulsar and Initial Spin of Neutron Star
}

\author{
Dong Lai \\ Theoretical Astrophysics, 130-33, California Institute of Technology \\ Pasadena, CA 91125 \\ E-mail: dong@tapir.caltech.edu
}

\begin{abstract}
Recent timing observations of PSR J0045-7319 reveal that the neutron star/B star binary orbit is decaying on a time scale of $\left|P_{\text {orb }} / \dot{P}_{\text {orb }}\right|=0.5 \mathrm{Myr}$, shorter than the characteristic age $\left(\tau_{c}=3 \mathrm{Myr}\right)$ of the pulsar (Kaspi et al. 1996a). We study mechanisms for the orbital decay. The standard weak friction theory based on static tide requires far too short a viscous time to explain the observed $\dot{P}_{\text {orb }}$. We show that dynamical tidal excitation of g-modes in the B star can be responsible for the orbital decay. However, to explain the observed short decay timescale, the B star must have some significant retrograde rotation with respect to the orbit - The retrograde rotation brings lower-order g-modes, which couple much more strongly to the tidal potential, into closer "resonances" with the orbital motion, thus significantly enhancing the dynamical tide. A much less likely possibility is that the g-mode damping time is much shorter than the ordinary radiative damping time. The observed orbital decay timescale combined with a generic orbital evolution model based on dynamical tide can be used as a "timer", giving an upper limit of 1.4 Myr for the age of the binary system since the neutron star formation. Thus the characteristic age of the pulsar is not a good age indicator. Assuming standard magnetic dipole braking for the pulsar and no significant magnetic field decay on a timescale $\lesssim 1 \mathrm{Myr}$, the upper limit for the age implies that the initial spin of the neutron star at birth was close to its current value.
\end{abstract}

Subject headings: binaries: close - pulsars: individual (PSR J0045-7319) - stars: neutron - stars: oscillations - stars: rotation - hydrodynamics - supernovae

\section{Introduction}

One of the fundamental questions in the study of pulsars concerns the initial conditions of neutron stars at birth. In particular, the initial spin of neutron star is related to such issues as angular momentum coupling in the progenitor stars, supernova explosion mechanisms and 
gravitational wave generation from core collapse and nascent neutron stars. Unfortunately, except for the Crab pulsar, for which the initial spin can be directly inferred from the measured pulsar period $P_{p}$ and its time-derivatives $\dot{P}_{p}, \ddot{P}_{p}$ and the known age, our knowledge about this quantity is rather limited. Statistical studies of the evolution of pulsar population (e.g., Narayan 1987), the energetics of pulsar nebulae (Helfand \& Becker 1987) and possible old pulsar/supernova remnant associations (Kaspi et al. 1996b) give some indications that neutron stars are formed rotating at a moderate spin period $10-100 \mathrm{~ms}$. However, all these methods suffer from uncertainties, and other independent constraints on the initial spins of neutron stars are highly valuable.

The PSR J0045-7319 binary contains a radio pulsar $\left(P_{p}=0.93 \mathrm{~s}\right)$ and massive B-star companion in an eccentric, 51.17 days orbit (Kaspi et al 1994). This system has recently been shown to exhibit spin-orbit precessions due to the rapid, misaligned rotation of the B star, which strongly suggests that the neutron star received a kick at birth from asymmetric supernova (Lai, Bildsten \& Kaspi 1995, Kaspi et al. 1996a). Recent timing observations also reveal that the orbit is decaying on a time scale $\left|P_{\text {orb }} / \dot{P}_{\text {orb }}\right|=0.5 \mathrm{Myr}$, shorter than the pulsar's characteristic age $\tau_{c}=P_{p} /\left(2 \dot{P}_{p}\right)=3 \mathrm{Myr}$ (Kaspi et al. 1996a). In this paper, we study the physical mechanisms for the rapid orbital decay, and demonstrate the potential of using the orbital decay time to constrain the age and initial spin of the pulsar.

The fiducial numbers we adopt for the current PSR J0045-7319 system are: pulsar mass $M_{p}=1.4 M_{\odot}$, companion mass $M_{c}=8.8 M_{\odot}$, radius $R_{c}=6.4 R_{\odot}$, and orbital period $P_{\text {orb }}=51.17$ days, semi-major axis $a=20 R_{c}$, eccentricity $e=0.808$ (Kaspi et al. 1996a, Bell et al 1995).

\section{Mechanisms for the Orbital Decay}

Since the mass loss from the B star is completely negligible (Kaspi et al. 1996a), tidal interaction is the most natural cause for the orbital decay in the PSR J0045-7319 system. There are two kinds of tides: static and dynamical. The static tide corresponds to the global,

quadrupolar distortion of the star. In the presence of finite viscosity, a tidal lag angle $\theta_{\text {lag }} \propto t_{\text {visc }}^{-1}$ develops between the tidal bulge and the line joining the pulsar and the B star. The orbital evolution and spin evolution are driven by the viscous tidal torque $\sim\left(G M_{p}^{2} R_{c}^{5} / a^{6}\right) \theta_{\text {lag. However, }}$. this "weak friction theory" based on static tide cannot explain the orbital decay in the PSR J0045-7319 binary for two reasons: (i) Using the general tidal equations (e.g., Alexander 1973) together with constraints on the stellar rotation rate and spin-orbit inclination angle, we have found that a viscous time $t_{\text {visc }}$ of less than 30 days is needed in order to explain the observed $\dot{P}_{\text {orb }}$; Such a small viscous time is almost certainly impossible. (e.g., even if the star were completely convective, the viscous time would still be longer than one year). (ii) The same amount of torque that induces the orbital decay also acts on the B star; Since the stellar spin $S$ is much less than the orbital angular momentum $L$, the static tide would lead to rapid spin-orbit synchronization and alignment if it were to explain the observed $\dot{P}_{\text {orb }}$, in contradiction with observations. In the following, we discuss the physics and outline the main result of a dynamical tide theory that can 
explain the observations. A more complete analyses will be presented in a future paper.

At each periastron passage, the pulsar excites non-radial (mainly $l=2$ quadrupolar) oscillations in the B star, therefore energy and angular momentum are transferred between the orbit and the star. Write the Lagrangian displacement in the rotating frame of the star as $\vec{\xi}(\mathbf{r}, t)=\sum_{\alpha} a_{\alpha}(t) \vec{\xi}_{\alpha}(\mathbf{r})$, where $\vec{\xi}_{\alpha}(\mathbf{r})$ is the eigenmode, normalized via $\int d^{3} x \rho \vec{\xi}_{\alpha}^{*} \cdot \vec{\xi}_{\alpha}=1$, and $\alpha=\{n \lambda m\}$ is the mode index ( $n$ is the radial order of the mode, $\lambda$ and $m$ specify the angular eigenfunction; note that $\lambda=l$ only for nonrotating star). The mode amplitude $a_{\alpha}(t)$ is then governed by the equation

$$
\ddot{a}_{\alpha}+\omega_{\alpha}^{2} a_{\alpha}+2 \gamma_{\alpha} \dot{a}_{\alpha}=\frac{G M_{p} W_{l m} Q_{\alpha}}{D^{l+1}} e^{-i m\left(\Phi-\Omega_{s} t\right)},
$$

where $\omega_{\alpha}$ is the mode angular frequency (in the rotating frame), $\gamma_{\alpha}$ the amplitude damping rate, $D(t)$ is the orbital separation, $\Phi(t)$ the orbital phase, $\Omega_{s}$ is the rotation rate of the companion!, $W_{l m}$ is a constant as defined in Press and Teukolsky (1977), and $Q_{\alpha}$ is the tidal coupling coefficient as given by $Q_{\alpha}=\int d^{3} x \rho \overrightarrow{\xi_{\alpha}^{*}} \cdot \nabla\left(r^{l} Y_{l m}\right)$.

The energy transfer between the star and orbit during each periastron passage depends on the phases of the oscillation modes, thus in general varies from one passage to another (e.g., Kochanek 1992; Mardling 1995). An important measure of the strength of dynamical tide is the energy $\Delta E_{s}$ transferred to the star (and the corresponding angular momentum transfer $\Delta J_{s}$ ) during the "first" periastron when there is no oscillation present initially. This can be calculated using the formalism of Press and Teukolsky (1977), generalized to include stellar rotation and elliptic orbit. To the leading ( $l=2$ quadrupole) order, we have

$$
\Delta E_{s}=\frac{G M_{p}^{2}}{R_{c}}\left(\frac{R_{c}}{a_{p}}\right)^{6} T_{2}(\eta), \quad T_{2}(\eta) \simeq 2 \pi^{2} \sum_{\alpha} \frac{\left(Q_{\alpha} K_{2 m, \alpha}\right)^{2}}{F_{\alpha}},
$$

where $\eta=\left(M_{c} / M_{t}\right)^{1 / 2}\left(a_{p} / R_{c}\right)^{3 / 2}$ is the ratio of the time for periastron passage and the stellar dynamical time, $M_{t}=M_{p}+M_{c}$ is the total mass, and $a_{p}=a(1-e)$ is the periastron distance. The factor $F_{\alpha}=1-\left(i / \omega_{\alpha}\right) \int d^{3} x \rho \vec{\xi}_{\alpha}^{*} \cdot\left(\vec{\Omega}_{s} \times \vec{\xi}_{\alpha}\right)$ is of order unity, and $K_{l m, \alpha}$ depends on the orbital trajectory and the mode frequency:

$$
K_{l m, \alpha}=\frac{W_{l m}}{2 \pi} \int_{-P_{\mathrm{orb}} / 2}^{P_{\mathrm{orb}} / 2} d t \frac{1}{D^{l+1}} \cos \left[\omega_{\alpha} t+m \Phi(t)-m \Omega_{s} t\right]
$$

with the integration centered around periastron. We can obtain a similar expression for the angular momentum transfer:

$$
\Delta J_{s}=\frac{G M_{p}^{2}}{R_{c}}\left(\frac{R_{c}}{a_{p}}\right)^{6}\left(\frac{R_{c}^{3}}{G M_{c}}\right)^{1 / 2} S_{2}(\eta), \quad S_{2}(\eta) \simeq 2 \pi^{2} \sum_{\alpha}\left(\frac{-m}{\omega_{\alpha}}\right) \frac{\left(Q_{\alpha} K_{2 m, \alpha}\right)^{2}}{F_{\alpha}} .
$$

\footnotetext{
${ }^{1}$ The observation of orbital precession implies that the stellar spin and the orbital angular momentum are not aligned. Here we assume they are aligned for simplicity. Thus $\Omega_{s}$ should be understood as the component of the spin rate perpendicular to the orbital plane.
} 
Clearly, the most strongly excited modes which contribute most to the energy transfer are those (i) propagating in the same direction as the orbit (the $m=-2$ modes); (ii) having frequencies in the inertial frame $\left(\omega_{\alpha}-m \Omega_{s}\right) \sim\left(-m \dot{\Phi}_{p}\right)$, where $\dot{\Phi}_{p}=\Omega_{\text {orb }}(1+e)^{1 / 2} /(1-e)^{3 / 2}=2 \pi /(3.2$ days $)$ is the orbital frequency at periastron ("resonance" condition); and (iii) having relatively large $Q_{\alpha}$. For nonrotating star, these modes are g-modes of radial order $n=5-9$ (i.e., $g_{5}-g_{9}$ ).

The rapid rotation of the B star changes its g-modes significantly. Since the spin frequency is comparable to the relevant g-mode frequencies, a perturbation treatment of the rotation effect is not valid. Neglecting the centrifugal force, the g-modes of rotating star can be calculated using the "traditional approximation" (e.g., Unno et al. 1989), where the Coriolis force associated with the horizontal component of the spin angular velocity is ignored (valid because the horizontal displacement of the g-mode is larger than the radial displacement). We correct the g-mode frequencies of a $\Gamma=4 / 3, \Gamma_{1}=5 / 3$ nonrotating polytropef using the numerical result of Berthomieu et al. (1978) to obtain the mode frequencies of the rotating star. Since $\left|\omega_{\alpha} / \Omega_{s}\right|$ is greater than unity (although not much greater) for the modes that are strongly excited, the rotational corrections to the mode frequencies and wavefunctions are not large (less than $40 \%$ in $\left.\omega_{\alpha}\right)$. We therefore adopt $Q_{\alpha}$ to be approximately the same as that of the non-rotating star; its validity is also indicated by an asymptotic analysis (Rocca 1987).

Figure 1 shows the function $T_{2}(\eta)$ for different values of $\Omega_{s}$. We see that rotation can significantly change the strength of dynamical tide. In particular, at $\eta=7$ (appropriate for the current PSR J0045-7319 system), retrograde rotation $\left(\Omega_{s}<0\right)$ can increase $\Delta E_{s}$ from the nonrotating value by more than two orders of magnitude. Physically, this dramatic effect of rotation on the tidal strength comes about because (i) the retrograde rotation brings lower-order g-modes, which couple to the tidal potential more strongly, into closer "resonances" with the orbital motion; (ii) The tidal coupling coefficient $Q_{\alpha}$ depends very strongly on the order of the mode. For example, for $\hat{\Omega}_{s} \equiv \Omega_{s}\left(G M_{c} / R_{c}^{3}\right)^{-1 / 2}=-0.4$, the most strongly excited modes are $g_{4}-g_{5}$, while $g_{1}-g_{3}$ are also much more strongly excited compared to the nonrotating case. The function $S_{2}(\eta)$ has similar behavior as $T_{2}(\eta)$, and we find that typically $S_{2} \simeq 2 T_{2}$ to within $10 \%$ (for $\left.\left|\hat{\Omega}_{s}\right|<0.5\right)$.

The radiative damping times of f-mode and low-order g-modes of a massive main sequence star range from 10's to 100's of years (Saio \& Cox 1980; Dziembowski et al. 1993), much longer than $P_{\text {orb }}$. Figure 2 shows an example of the dynamical tidal evolution obtained by integrating Eq. (1) and the orbital equations. The average mode energy settles into a constant value $E_{s} \simeq \Delta E_{s}$ (a "fixed point" of the dynamical system) after a few damping times, and the orbital energy decreases secularly according to

$$
\dot{E}_{\text {orb }} \simeq-2 \gamma\left|\Delta E_{\text {orb }}\right|=-2 \gamma\left(\Delta E_{s}+\Omega_{s} \Delta J_{s}\right),
$$

\footnotetext{
${ }^{2}$ This is appropriate for a massive star, although the g-modes depend somewhat on the size of the convective core, and hence on the stellar age. We have tested that $\left|\Delta E_{s}\right|$ is rather insensitive to the core size.
} 
where $\gamma_{\alpha}=\gamma=1 / t_{\text {damp }}$ (i.e., we assume that the damping times are the same for different modes; this is approximately valid since only a small number of modes are strongly excited). With $S_{2} \simeq 2 T_{2}$, we have $\Delta J_{s} \simeq 2\left(R_{c}^{3} / G M_{c}\right)^{1 / 2} \Delta E_{s}$, and the secular orbital decay rate is then given by

$$
\frac{\dot{P}_{\text {orb }}}{P_{\text {orb }}} \simeq-\frac{1+2 \hat{\Omega}_{s}}{0.24 \mathrm{Myr}}\left(\frac{100 P_{\text {orb }}}{t_{\text {damp }}}\right)\left(\frac{T_{2}}{10^{-2}}\right) .
$$

For nonrotating star, we find $T_{2} \simeq 2.6 \times 10^{-4}$, thus a very short damping time $t_{\text {damp }} \simeq 5.5 P_{\text {orb }}$ is needed to explain the observed $\dot{P}_{\text {orb }}$. However, when the star has significant retrograde rotation, the observed orbital decay rate can be explained with ordinary radiative damping.

If the mode damping time is shorter than the orbital period, then the secular orbital decay rate should be given by $\dot{P}_{\text {orb }} \sim-\left|\Delta E_{\text {orb }}\right| /\left|E_{\text {orb }}\right|$. This would require significant enhancement to the mode dissipation compared to ordinary radiative damping. Nonlinear mode damping (Kumar \& Goodman 1996) is unimportant since the mode energy $E_{s} /\left(G M_{c}^{2} / R_{c}\right) \sim 10^{-7}\left(t_{\mathrm{damp}} / 100 P_{\mathrm{orb}}\right)$, implied from the observed orbital decay rate, is much smaller than the threshold $E_{s, t h} /\left(G M_{c}^{2} / R_{c}\right) \sim\left(\omega_{\alpha} t_{t h}\right)^{-0.4} \sim 10^{-3}$ needed for the three-mode parametric resonant coupling (where $t_{t h} \sim 10^{4} \mathrm{yr}$ is the thermal time of the star, and we have approximated the damping time of the $n \sim l>>1$ mode by $\left.t_{t h} / l^{8}\right)$. We consider this possibility rather unlikely.

\section{Orbital Decay Model and Constraints on the Pulsar Age and Initial Spin}

We now consider the long-term evolution of the binary system. Equations (2) and (5) provide a scaling relation for the orbital decay rate. Let $T_{2}(\eta) \propto \eta^{-4 \nu}$ (with $\nu \simeq 1,0.5,0.2$ for $\hat{\Omega}_{s}=0,-0.2,-0.4$ respectively), we obtain

$$
\dot{P}_{\text {orb }}=-A P_{\text {orb }}^{-7 / 3-4 \nu}(1-e)^{-6(1+\nu)},
$$

where the constant $A$ can be fixed by the observed current $\dot{P}_{\text {orb }}$ value. Using $\dot{L}=-\dot{J}_{s} \simeq-2\left(R_{c}^{3} / G M_{c}\right)^{1 / 2} \dot{E}_{s}$, we have

$$
\frac{\dot{L}}{L}=\frac{1}{\beta} \frac{P_{\text {orb }, 0}\left(1-e_{0}^{2}\right)^{1 / 2}}{P_{\text {orb }}\left(1-e^{2}\right)^{1 / 2}} \frac{2 \dot{P}_{\text {orb }}}{3 P_{\text {orb }}}
$$

where the subscript " 0 " indicates current values, and the parameter $\beta \simeq 50\left(1+2 \hat{\Omega}_{s}\right)$ ranges from 10 to 50 for $-0.4<\hat{\Omega}_{s}<0$. Note that the long-term orbital angular momentum change $]^{3}$ is much less than the change in the orbital energy since

\footnotetext{
${ }^{3}$ The orbital angular momentum is transferred into the spin, whose evolution can be easily evaluated: Since $\dot{\Omega}_{s} / \Omega_{s} \simeq\left(0.04 / \hat{\Omega}_{s}\right)\left(\dot{P}_{\text {orb }} / P_{\text {orb }}\right)$ (with the moment of inertia $\left.0.1 M_{c} R_{c}^{2}\right)$, the timescale for changing the stellar spin (both its magnitude and direction) is longer than the orbital decay timescale. This feature is qualitatively different from static tide.
} 
$\left|\Delta J_{s} / L\right| \simeq\left|\Delta E_{s} / E_{\text {orb }}\right| \Omega_{\text {orb }}\left(G M_{c} / R_{c}^{3}\right)^{-1 / 2}\left(1-e^{2}\right)^{-1 / 2}<<\left|\Delta E_{s} / E_{\text {orb }}\right|$. The evolution of eccentricity is given by

$$
\dot{e}=\frac{1-e^{2}}{e}\left(\frac{\dot{P}_{\text {orb }}}{3 P_{\text {orb }}}-\frac{\dot{L}}{L}\right) .
$$

For the current PSR J0045-7319 binary, we find $\dot{e} \simeq 10^{-14} \mathrm{~s}^{-1}$, consistent with the observational upper limit for $\dot{e}$ (Kaspi et al. 1996a).

Equations (7)-(9) can be integrated backward to give $P_{\text {orb }}$ and $e$ at earlier timest The parameters $\nu$ and $\beta$ have been chosen to reflect their allowed ranges. The results are shown in Figure 3. We see that $e \rightarrow 1$ and $P_{\text {orb }} \rightarrow \infty$ as $(-t)$ approaches a fixed value $\lesssim 1.4 \mathrm{Myr}$, independent of the parameters $\nu$ and $\beta$. This feature can be understood as follows: Since the angular momentum transfer in a periastron passage is relatively small compared to the energy transfer, the periastron distance $a_{p}$ and hence $\Delta E_{s}$ remain approximately constant during the evolution. Thus $\left|\dot{P}_{\text {orb }} / P_{\text {orb }}\right| \propto \Delta E_{s} /\left|E_{\text {orb }}\right|$ is larger at earlier times.

The observed $\dot{P}_{\text {orb }}$ and our generic orbital evolution model therefore set an upper limit $\simeq 1.4$ Myr to the age of the binary system since the last supernova . This is significantly smaller than the characteristic age $\tau_{c}$ of the pulsar, implying that the latter is not a good age indicator. In principle, the discrepancy between the age and $\tau_{c}$ may be explained in several ways: (i) The pulsar has been slowed down by an earlier phase of mass accretion; (ii) The pulsar braking index is much larger than 3 (the canonical dipole value); (iii) The pulsar magnetic field decays on a timescale $\lesssim 1$ Myr; (iv) The initial spin period of the pulsar is not much shorter than the current value. The extremely small mass loss from the B star (Kaspi et al. 1996a) argues strongly against (i). The observed braking indices measured for three of the youngest pulsars range from 2.0 to 2.8 , and in view of the statistical evidence and physical argument against rapid field decay, we consider (ii) and (iii) to be rather unlikely. Using the standard magnetic dipole braking for the pulsar spin-down, the constraint on the pulsar age implies that the initial spin period of the pulsar is longer than 0.7 second, very close to its current value. Thus PSR J0045-7319 was formed rotating very slowly, and its progenitor must also have rather slow rotation. We note that such a small initial spin rate of the pulsar may result from the random torque on the nascent neutron star due to asymmetry in the supernova (e.g., Burrows et al. 1995).

\footnotetext{
${ }^{4}$ We assume there is no major structure change in the B star during the orbital evolution. This is most likely to be valid because the Kelvin-Holmholtz timescale $\left(\sim 10^{4}\right.$ years $)$ is much shorter than the orbital evolution time.

${ }^{5}$ In the unlikely case of $t_{\text {damp }} \lesssim P_{\text {orb }}$, the scaling relation for $\dot{P}_{\text {orb }}$ is modified from Eq. (7) to $\dot{P}_{\text {orb }} \propto$ $-P_{\text {orb }}^{-10 / 3-4 \nu}(1-e)^{-6(1+\nu)}$, and an absolute upper limit to the age cannot be obtained (see the dotted line in Fig. 3). However, the age can still be constrained since the probability of forming a highly eccentric, bound orbit after supernova is small.
} 


\section{Discussion}

Our analyses of PSR J0045-7319 binary orbital decay show that the B star companion is most likely to have a retrograde rotation with respect to the orbit. Since the spin of the B star is expected to have been aligned with the orbital angular momentum before the supernova, the current misaligned configuration could have come about only if the neutron star received a kick at birth due to asymmetry in the supernova. The kick velocity must (i) have had a component out of the original orbital plane in order to misalign $\mathbf{L}$ and $\mathbf{S}$; (ii) have had a significant component in the direction opposite to the orbital velocity of the progenitor in order to reverse $\mathbf{L}$. The current timing data yield two degenerate solutions for the range of the spin-orbit inclination angle, allowing for both prograde and retrograde rotation (Kaspi et al. 1996a). Long-term (i.e., some fraction of the precession period $\sim 500$ years) timing observation should distinguish these two

possibilities. Dedicated optical observation of the companion may also give useful constraints on the excited modes (Kumar et al. 1995).

Our analyses have also demonstrated that the orbital decay can be used to put concrete constraints on the pulsar age and initial spin. The tide-induced orbital decay of the PSR B1259-63/Be star binary (the only other known radio pulsar/main sequence star binary) is too slow to be observable owing to its larger orbital separation at periastron. Finding more systems similar to PSR J0045-7319 will allow for determination of systematic constraints on the physical conditions of neutron stars at birth and supernova characteristics.

I thank Vicky Kaspi for sharing the observational data with me and helpful discussion and comments. I also thank Lars Bildsten, Peter Goldreich, Norm Murray and Yanqin Wu for useful discussion. This research is supported by the Richard C. Tolman Fellowship at Caltech, NASA Grant NAG 5-2756, and NSF Grant AST-9417371.

\section{REFERENCES}

Alexander, M. E. 1973, Astrophys. and Space Sci., 23, 459.

Bell, J. F., Bessell, M. S., Stappers, B. W., Bailes, M. \& Kaspi, V. M. 1995, ApJ, 447, L117

Berthomieu, G., et al. 1978, A\&A, 70, 596.

Burrows, A., Hayes, J., \& Fryxell, B. A. 1995, ApJ, 450, 830.

Dziembowski, W. A., Moskalik, P., \& Pamyatnykh, A. A. 1993, MNRAS, 265, 588.

Helfand, D. J., \& Becker, R. H. 1987, ApJ, 314, 203.

Kaspi, V. M., et al. 1994, ApJ, 423, L43 
Kaspi, V. M., Bailes, M., Manchester, R. N., Stappers, B. W., \& Bell, J. F. 1996a, Nature (in press).

Kaspi, V. M., et al. 1996b, AJ, in press.

Kochanek, C. S. 1992, ApJ, 385, 604.

Kumar, P., Ao, C. O., \& Quataert, E. J. 1995, ApJ, 449, 294

Kumar, P., \& Goodman, J. 1996, ApJ, in press

Lai, D., Bildsten, L., and Kaspi, V. M. 1995, ApJ, 452, 819

Mardling, R. A. 1995, ApJ, 450, 732.

Narayan, R. 1987, in "The origin and Evolution of Neutron Stars", ed. D. J. Helfand \& J.-H. Huang (D. Reidel Pub.: Dordrecht).

Press, W. H., \& Teukolsky, S. A. 1977, ApJ, 213, 183

Rocca, A. 1987, A\&A, 175, 81.

Saio, H., \& Cox, J. P. 1980, ApJ, 236, 549.

Unno, W., Osaki, Y., Ando, H., Saio, H., \& Shibahashi, H. 1989, Nonradial Oscillations of Stars (Univ. of Tokyo Press) 
Fig. 1. - The function $T_{2}(\eta)$ (defined in Eq. [2]) for different rotation rates: $\hat{\Omega}_{s}=0$ (solid lines), 0.2 (dotted lines), -0.2 (short-dashed lines), and -0.4 (long-dashed lines). Negative $\hat{\Omega}_{s}$ corresponds to retrograde rotation. The heavy lines are for elliptic orbit with $e=0.808$ (the current value for the PSR J0045-7319 system), and the light lines for parabolic orbit.

Fig. 2.- Evolution of $\delta E_{\text {orb }} \equiv E_{\text {orb }}-E_{\text {orb }, 0}$ and $E_{s}$ (mode energy in the rotating frame) due to dynamical tide (with $\hat{\Omega}_{s}=-0.2$ ). Only the $g_{3}-g_{8}$ modes are included in the calculation, and relatively large damping rates are chosen for clearer illustration: $\gamma=0.1 / P_{\text {orb }}$ (solid curves) and $\gamma=0.05 / P_{\text {orb }}$ (dotted curves).

Fig. 3. - The long-term (backward) evolution of the binary period and eccentricity $(t=-3$ Myr corresponds to the pulsar's characteristic age). The solid lines are for $\beta=10$, with $\nu=0.2,0.5,1$ (left to right), the short-dashed lines are for $(\nu, \beta)=(0.5,20)$, the long-dashed lines for $(\nu, \beta)=(0.5,50)$. The dotted curves are for a model assuming $t_{\text {damp }} \lesssim P_{\text {orb }}$ with $(\nu, \beta)=(1,50)$ (see footnote 5 ). 


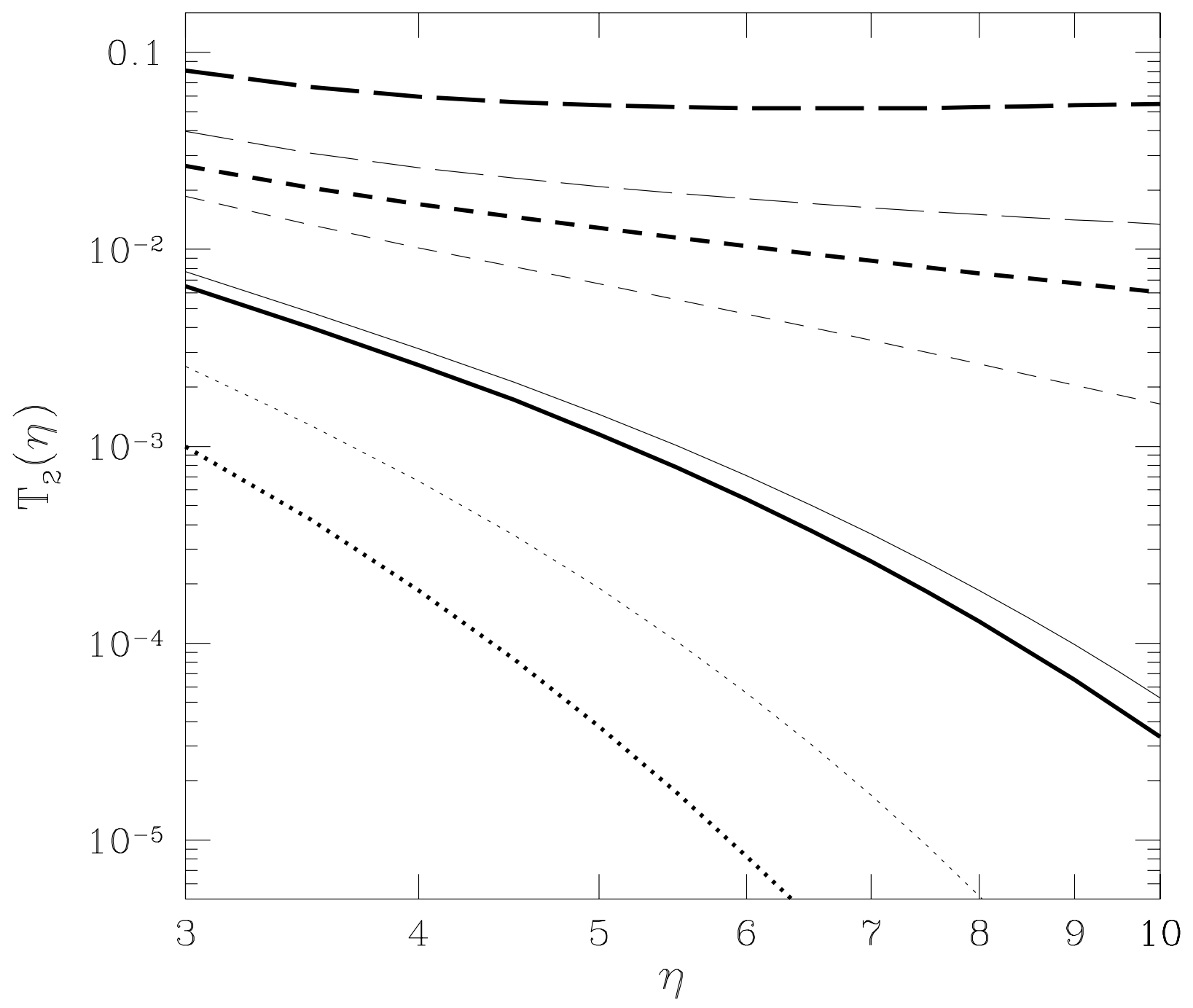

Fig. 1 


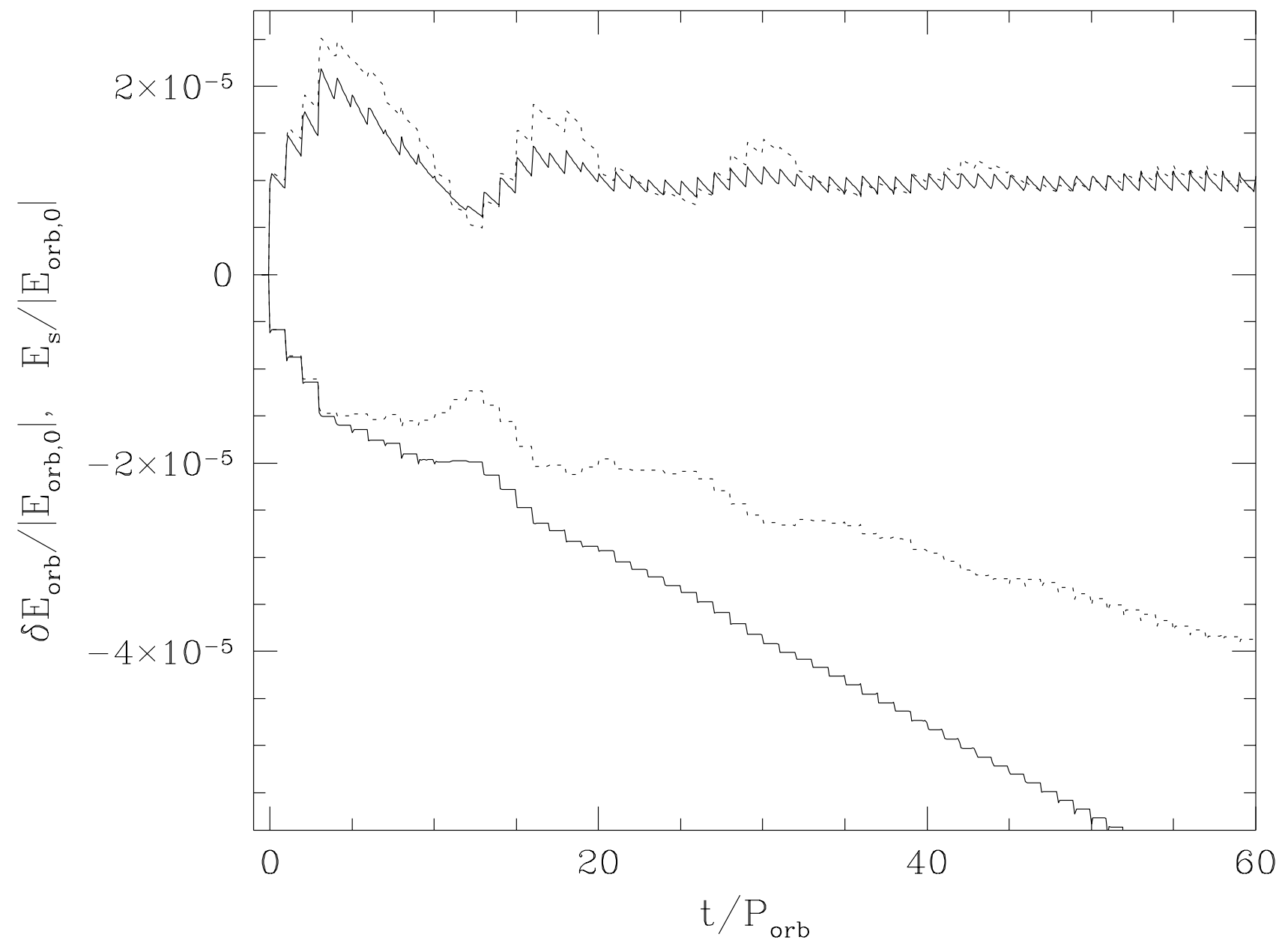

Fig.2 


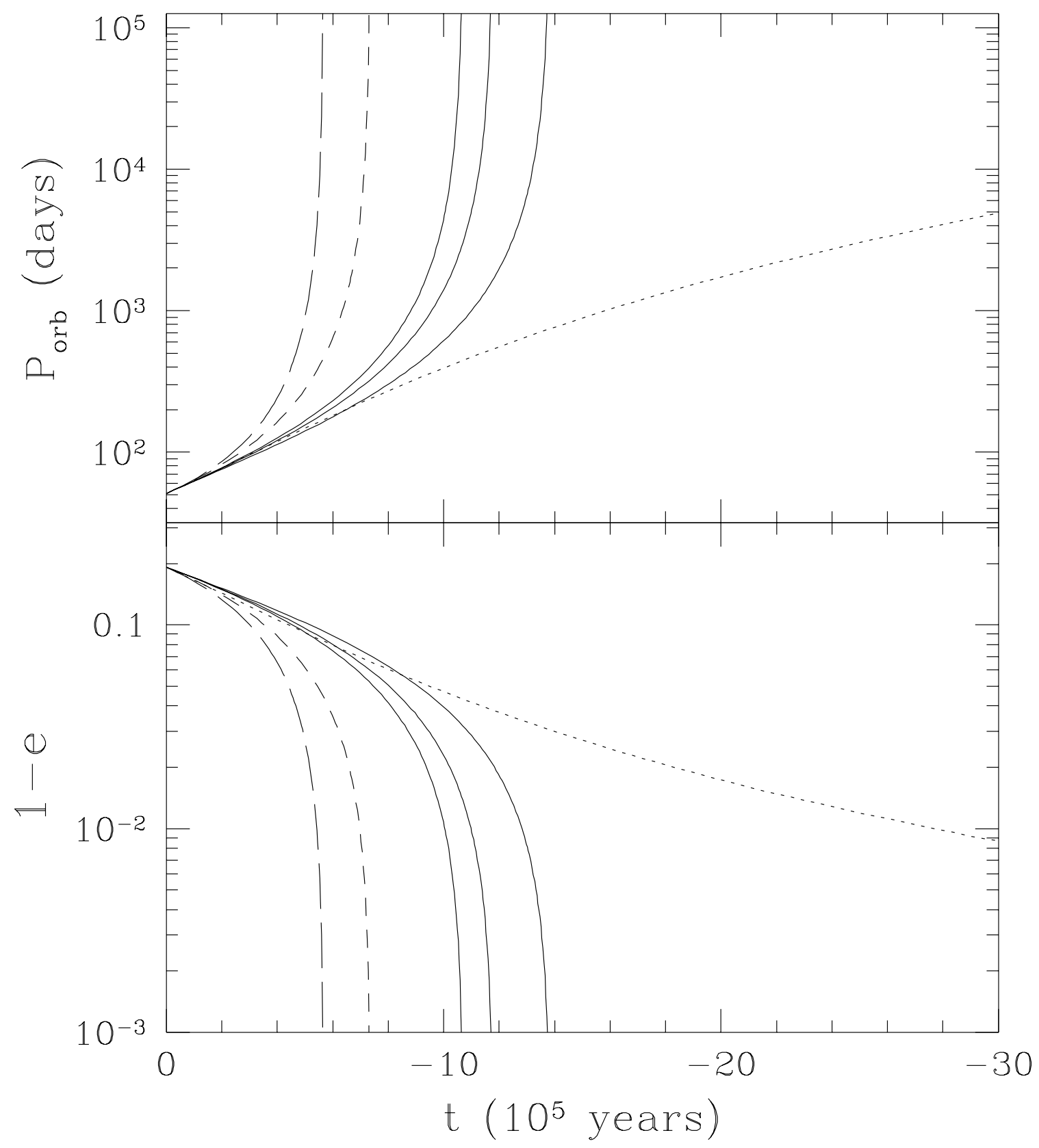

Fig. 3 